\title{
Limbic Activation and Psychophysiologic Responses to Aversive Visual Stimuli: Interaction with Cognitive Task
}

Israel Liberzon, M.D., Stephan F. Taylor, M.D., Lorraine M. Fig, M.B., Ch.B., Laura R. Decker, B.Sc., Robert A. Koeppe, Ph.D., and Satoshi Minoshima, M.D., Ph.D.

We mapped regional brain activity and peripheral psychophysiologic responses, occurring in response to evocative emotional stimuli, and examined whether task instructions could modulate limbic activation. Ten subjects viewed pictures with neutral or aversive emotional content during simultaneous measurement of peripheral psychophysiology and brain activity with $\left[{ }^{15}\right.$ Olwater positron emission tomography (PET). Cognitive task was manipulated by having the subjects rate the pictures or perform a recognition memory task. Aversive pictures, relative to neutral pictures, increased cerebral activity in bilateral amygdala, thalamic/hypothalamic area, midbrain, and left lateral prefrontal cortex, along with greater skin conductance responses (SCR). Voxel-by-voxel correlation coefficients between regional brain activity and SCR showed significant positive correlation peaks in the thalamus and right amygdala. Limbic activation was significantly greater during the rating condition compared to the recognition condition, suggesting that when task demands modify emotional responses, this modulation can occur at the level of limbic activity.

[Neuropsychopharmacology 23:508-516, 2000] ( 2000 American College of Neuropsychopharmacology. Published by Elsevier Science Inc.
KEY WORDS: PET; rCBF; Amygdala; Skin conductance; Recognition

Emotional responses are regulated by limbic regions of the brain, such as the amygdala, hypothalamus, limbic midbrain, and limbic cortex (anterior cingulate, orbitofrontal, temporal polar, medial temporal). The cytologi-

From the Department of Psychiatry (IL, SFT, LRD) and Department of Internal Medicine, Division of Nuclear Medicine (RAK, SM), University of Michigan, Ann Arbor, MI; and the Department of Psychiatry (IL) and Department of Nuclear Medicine Services (LMF), Veterans Administration Medical Center, Ann Arbor, MI.

Address correspondence to: Israel Liberzon, M.D., Psychiatry / PCT 116A, Veterans Affairs Medical Center, 215 Fuller Road, Ann Arbor, MI 48105.

Received 17 January 2000; revised 27 April 2000; accepted 1 June 2000. cal organization of various limbic structures and their inter-connectivity, suggest that these regions comprise a functional network (Alheid and Heimer 1988). Extracellular recording, electrical stimulation and lesion studies implicate the amygdala and related limbic structures in the expression or modulation of emotional responses (Adolphs et al. 1995; LeDoux 1986; McGaugh et al. 1996). Animal and human studies suggest that these same regions modulate autonomic components of emotional responses (Bechara et al. 1995; LaBar et al. 1995; Mangina and Beuzeron-Mangina 1996). Recent functional neuroimaging studies have demonstrated that the amygdala and cortico-limbic structures respond to fearful/aversive stimuli (Irwin et al. 1996; Reiman et al. 1997; Taylor et al. 1998; LaBar et al. 1998; Morris et al. 1999a), perception of facial expressions of fear and disgust (Breiter et al. 1996; Phillips et al. 1997; Whalen et al. 1998), perception of linguistic threat (Isen- 
berg et al. 1999) and of non verbal emotional vocalization (Morris et al. 1999b).

Emotional responses are highly variable and influenced by diverse factors such as arousal, temperament, prior expectation, context and task demands - factors which may affect a controlled experiment. For example, the skin conductance response (SCR) to a social scene will vary depending upon the narrative context in which the scene is viewed (Geen and Rakosky 1973; Lazarus 1984). In a study examining the effects of emotional stimulus content on memory, we noted that amygdaloid activation disappeared when the task changed from rating stimuli to recognizing them, suggesting an effect of task instruction on emotional processing (Taylor et al. 1998). Although our finding was confounded by an order effect, the modulation of emotional responses by cognitive states has been demonstrated in numerous behavioral studies (Folkman and Lazarus 1985; Smith et al. 1993). They were also consistent with observations by Lane and colleagues (1997) that reported a differential pattern of limbic activation when emotional responses were evoked during different cognitive task. Reiman and colleagues (1997) findings of different regional cerebral blood flow (rCBF) patterns associated with memory-generated vs. filmgenerated sadness, also support the notion of cognitive states modulating emotional responses. In this new study, we sought to investigate a hypothesis about one cognitive factor and how it might influence limbic activation.

The current $\left[{ }^{15} \mathrm{O}\right]$ water positron emission tomography (PET) study was designed to identify components of the limbic brain (extended amygdala, thalamus, hypothalamus) associated with an emotional and psychophysiologic response to aversive pictures. As converging validation, we also simultaneously recorded peripheral autonomic measures of emotional responses (Venables and Martin 1967) and applied a voxel-by-voxel correlation analysis in order to identify regions of the brain controlling these peripheral responses. To examine whether modification of task instructions would attenuate the activity in this network, we manipulated task instructions by having subjects either rate pictures in some scans or recognize pictures in other scans, predicting that when task instructions shifted from assessing emotional content to recognizing pictures, activation of central limbic regions would be attenuated.

\section{METHODS}

\section{Subjects}

Ten healthy female volunteers, were recruited from community advertisements (mean age $=26.1$, range: 19-44). Subjects had no past or current history of chronic medical or neurological illness. Absence of any Axis I psychiatric disorder was verified by a structured clinical interview (Spitzer et al. 1988). Because greater autonomic responses (Kring and Gordon 1998) and regional CBF differences (Gur et al. In press) have been documented in females, we studied only female subjects to maximize our ability to detect significant effects. All subjects gave written, informed consent after explanation of the experimental protocol, as approved by the local Institutional Review Board.

\section{Task Design}

The experimental design consisted of two orthogonal factors (stimulus valence and task), each with two levels (negative and neutral, rating and recognizing, respectively). During the PET scans, subjects viewed pictures from one of two sets, selected from the standardized International Affective Picture System (IAPS) (Lang and Greenwald 1988) and supplemented from our own collection (approximately 30\%). For the supplemental pictures, valence ratings obtained in a separate experiment were comparable to ratings from the IAPS (Taylor et al. unpublished data). We selected negative pictures which elicited emotional responses of fear/disgust, such as graphic pictures of facial mutilation, wounds, dead bodies, etc. Neutral pictures were selected which did not elicit strong emotions, positive or negative, such as people at rest, faces with neutral expressions, and benign scenes. Since processing of faces recruits specific brain regions (Dolan et al. 1996; Haxby et al. 1996), we matched the neutral and negative pictures with respect to the number of slides portraying faces and human figures.

The tasks consisted either of the subjects rating the pictures for aversive content (rating) or determining whether the pictures had been previously presented (recognition). In the rating phase, subjects received instructions to rate each picture verbally on a 5-point scale $(5=$ extremely disgusting and $1=$ not at all disgusting), indicating the degree to which they found the image disgusting. Within the rating phase, all pictures were novel. In the recognition phase, subjects were instructed that some slides would be repeated from the previous blocks, and they were to verbally indicate whether or not they recognized the slide, using a 3-point scale: "1" for previously seen; " 3 " for uncertainty; and " 5 " for not previously seen. Approximately $50 \%$ of the slides in the recognition blocks were repeated from the rating phase. During the rating phase the subjects were not told that pictures would later be repeated in a recognition task. The pictures ( $35 \mathrm{~mm}$ slides) were presented on a back-projecting slide carrousel (Kodak Model 570AF), on a screen suspended $40 \mathrm{~cm}$ from the subject, subtending 15 degrees of viewing angle. View- 
ing duration was $5 \mathrm{sec}$ for each slide, with no interstimulus interval.

Each subject saw 10 blocks of pictures and the stimuli presented in each block were either all negative slides or all neutral slides. The first two blocks consisted of the rating task, but without actual image acquisition, allowing the subjects to become comfortable with the scanner. Eight actual PET scans were acquired for each subject, beginning with the third block and continuing through the tenth and final block of pictures. The first two PET scans consisted of rating followed by four scans on the recognition task, and then two scans in the rating task.

\section{Data Acquisition}

Psychophysiological data were recorded using an MP100 psychophysiological monitoring system (BioPac Systems, Santa Barbara, CA). Skin conductance was recorded using $\mathrm{Ag} / \mathrm{AgCl}$ electrodes filled with isotonic $\mathrm{NaCl}$ unibase electrolyte, attached to the volar surface of the second phalanx of the first and third fingers of the left hand. Heart rate (HR) was monitored via EKG with an automated R-wave detector and filtered with a 60-Hz notch filter.

The subjects underwent PET scans in a Siemens CTI 931-08/12 scanner (CTI Inc., Knoxville, TN) which acquires 15 slices over an axial length of approximately 10 $\mathrm{cm}$. Before emission scanning, subjects had a transmission scan measured by external ${ }^{68} \mathrm{Ge} /{ }^{68} \mathrm{Ga}$ ring sources. For each emission scan, subjects were given an intravenous (i.v.) bolus injection of $50 \mathrm{mCi}$ of $\left[{ }^{15} \mathrm{O} \mathrm{H}_{2} \mathrm{O}\right.$. Data were collected in a single $60 \mathrm{sec}$ frame beginning $5 \mathrm{sec}$ after the arrival of the radioactivity in the brain. Lights were dimmed and ambient noise (from cooling fans) was minimal. Eight PET scans separated by 12 minutes were acquired for each subject. Stimuli presentation began 5 sec prior to image acquisition, and continued for the first $30 \mathrm{sec}$ of each scan (uptake phase of the tracer), so as to maximize changes in the PET signals associated with the task of interest (Cherry et al. 1993). The second $30 \mathrm{sec}$ of each scan always contained neutral stimuli, and subjects continued the task begun $35 \mathrm{sec}$ prior.

\section{Data Analysis}

Analysis of the PET data first employed a standardization process for each image that enabled averaging of image data both within and across subjects. Automated routines proportionally normalized images to the mean of global activity (arbitrarily set to 1000), and individual images within each subject were co-registered to the fourth scan to correct for head movement occurring between scans (usually less than $2 \mathrm{~mm}$ translation and less than 2 degrees rotation). A non-linear transformation was then applied to each image to standardize individual anatomy according to a reference brain atlas (Minoshima et al. 1992, 1993, 1994). Standardized images were smoothed with a 3D Gaussian filter, yielding a final, effective FWHM of around $14 \mathrm{~mm}$. Difference images were made by subtracting the scan obtained in one condition from that obtained in another condition. Mean differences were expressed as Z-scores, using the between-subjects variance (Friston et al. 1991; Worsley et al. 1992).

We performed analyses of negative versus neutral in each phase (rating and recognition) and analysis of the interaction of emotional content and task context, which we analyzed as a compound difference image, i.e., rating (negative minus neutral) minus recognition (negative minus neutral).

Planned or "A Priori" Analysis. We searched for significant activations on the basis of regional a priori hypotheses as well as an image-wide search unconstrained by regional predictions. For the a priori search region, we delimited a $120 \mathrm{cc}$ volume of brain in the following areas: medial frontal cortex (including rostral anterior cingulate gyrus), bilateral amygdaloid area, hypothalamus, and thalamus. Within this volume, a significance threshold of $Z=3.6$ yielded a false positive expectation of 0.05 (Worsley et al. 1992). We accepted $3.6>Z>3.09$ as trend-level activation $(p<.001$, uncorrected) in the region of interest, in order to permit comparisons with other published work. We also performed targeted volume of interest (VOI) analyses on bilateral amygdaloid complexes by placement of a 15.75 $\mathrm{mm}$ sphere at the stereotactic coordinates of the amygdala $(\mathrm{x}, \mathrm{y}, \mathrm{z}= \pm 24,-4,-16)$ in the Talairach and Tournoux atlas (Talairach and Tournoux 1988).

Since the diameter of the amygdala is closer to 10 $\mathrm{mm}$, we selected a slightly larger region to capture the individual variation in amgydala size and location, not corrected for by our warping routines. From our first study (Taylor et al. 1998), we also tested a VOI in the lingual gyrus (13.5 $\mathrm{mm}$ sphere; $\mathrm{x}, \mathrm{y}, \mathrm{z}=-12,-87,-7$ ). For our image-wide search, we reported activation foci that exceeded a corrected probability of $p<.05$, generally corresponding to a Z-score of around 4.4 for our image sets (Worsley et al. 1992). Six out of eighty individual scans were not analyzable due to technical difficulties (stimulus presentation or tracer injection), without affecting the total number of subjects or conditions analyzed.

Psychophysiological Correlation. To identify possible neural substrates of the peripheral SCR, we performed a voxel-by-voxel, within-subjects correlation analysis (Minoshima et al. 1995). For each voxel within each subject, we calculated a correlation coefficient between normalized pixel counts and the integrated SCR for that scan, for a total of eight observation pairs.

The estimated correlation coefficients were then transformed to Z-values using Fisher's Z-transforma- 
tion. The transformed maps were averaged across subjects, which further assured a normal distribution of the final summed correlation Z-map and increased the sensitivity of the analysis. Based on the theory of Gaussian random fields (Worsley et al. 1992), we determined a statistical threshold for the final correlation map. Pixelby-pixel correlation maps were analyzed with the same a priori defined ROIs, used in the analysis of activation peaks. This analysis has the advantage of providing good protection against outlier values leveraging correlation coefficients and causing false positives in a statistical parametric map.

Psychophysiological Data. The changes in skin conductance for each block of pictures were analyzed by analysis of variance of the peak conductance change as well as the conductance level integrated over the first 30 sec epoch of image presentation (relative to a $5 \mathrm{sec}$ baseline prior to image presentation). Heart rate was calculated beat-to-beat and averaged over this interval. Analysis of psychophysiology data was performed offline with AcKnowledge software (BioPac Systems, Santa Barbara, CA). Statistical analysis outside of custom-written software was performed with StatView (Abacus Concepts, Berkeley, CA).

\section{RESULTS}

\section{Behavioral and Psychophysiological Results}

Our subjects experienced the negative pictures as significantly more disturbing than the neutral pictures $(\mathrm{t}=$ $19.5, \mathrm{df}=9, p<.0001$ ) (Table 1). During the recognition phase, subjects demonstrated non-significantly greater accuracy in the classification of the negative pictures $(81 \pm 12 \%$ correct \pm S.D. for neutral, and $88 \pm 6.0 \%$ for negative pictures; $\mathrm{t}=1.7, \mathrm{df}=9, p=.12$ ).
Changes in skin conductance confirmed the differential emotional response to the pictures. While there was no difference in baseline measures of skin conductance prior to stimuli presentation, the negative stimuli elicited a larger maximal increase in SCR as compared to neutral stimuli (Rating, negative vs. neutral: $1.03 \pm 0.58$ vs. $0.52 \pm 0.36$; Recognition, negative vs. neutral: $0.96 \pm$ 0.80 vs. $0.81 \pm 0.56, \mu$ mho \pm S.D.; Effect of valence: $F$ $[1,9]=9.76, p=.01)$. There was a significant interaction of stimulus type by condition, such that the effect of negative pictures during rating was greater than the effect of negative pictures during recognition for conductance peak and integral $(\mathrm{F}[1,9]=10.21, p=.01 ; \mathrm{F}[1,9]=5.46, p=.04$, respectively). We found no effects - main or interaction - for stimulus type and phase on heart rate.

\section{Neuroimaging Results}

Activation Peaks. We examined the effects of negative versus neutral content of the stimuli during both the rating and recognition phases. During the rating phase, a significant activation focus in our area of interest centered on the limbic diencephalon, contiguous with the mid-thalamic nuclei and extended amygdala. A trend-level focus in the midbrain also appeared, just outside our area of interest, but continuous with the diencephalic focus (Figure 1). A volume of interest analysis with spherical VOIs placed at the stereotactic coordinates of the amygdala confirmed activation in the right and left amygdaloid regions $(\mathrm{t}=2.16, \mathrm{df}=9, p=.03$; $\mathrm{t}=2.21, \mathrm{df}=9, p=.03$, respectively) (see Figure 2).

In addition to the limbic activation, we also observed trend-level activation of the left middle frontal gyrus, as well as significant relative decreases in activity in the striate cortex. Trends to relative decrease were also noted in the lingual gyrus, orbital gyrus, and left cerebellum. During the recognition phase, negative emotional content produced significant activation in the

Table 1. Subjective and Psychophysiological Responses to Aversive Stimuli

\begin{tabular}{lccccc}
\hline & \multicolumn{2}{c}{ Rating } & & \multicolumn{2}{c}{ Recognition } \\
\cline { 2 - 3 } \cline { 5 - 6 } Parameter & Neutral & Negative & & Neutral & Negative \\
\hline $\begin{array}{l}\text { Subject responses } \\
\quad \text { Ratings of disgust }\end{array}$ & $1.0 \pm 0.02$ & $3.6 \pm 0.46^{a}$ & & - & - \\
$\quad \begin{array}{l}\text { Recognition accuracy } \\
\text { Psychophysiological measurements }\end{array}$ & & & $81 \pm 12 \%$ & $88 \pm 6$ \\
$\quad$ Skin conductance & & & & \\
$\quad$ Baseline & $5.19 \pm 2.01$ & $5.10 \pm 1.77$ & & $5.47 \pm 1.92$ & $4.89 \pm 1$ \\
$\quad$ Max amplitude increase & $0.52 \pm 0.36$ & $1.03 \pm 0.58^{\mathrm{b}, \mathrm{d}}$ & & $0.81 \pm 0.56$ & $0.96 \pm 0$ \\
$\quad$ Integrated conductance & $10.06 \pm 11.59$ & $23.44 \pm 16.55^{\mathrm{c}, \mathrm{d}}$ & $16.99 \pm 15.7$ & $23.1 \pm 2$ \\
$\quad$ Heart Rate & $73.8 \pm 14.2$ & $74.6 \pm 15.4$ & & $73.1 \pm 12.8$ & $74.8 \pm 1$ \\
\hline
\end{tabular}

\footnotetext{
${ }^{a}$ Negative greater than neutral, $p<.0001$

${ }^{b}$ Negative greater than neutral, $p<.005$.

${ }^{c}$ Negative greater than neutral, $p<.05$.

${ }^{d}$ Effect of negative $=$ neutral during encoding greater than during recognition, $p<.04$.
} 


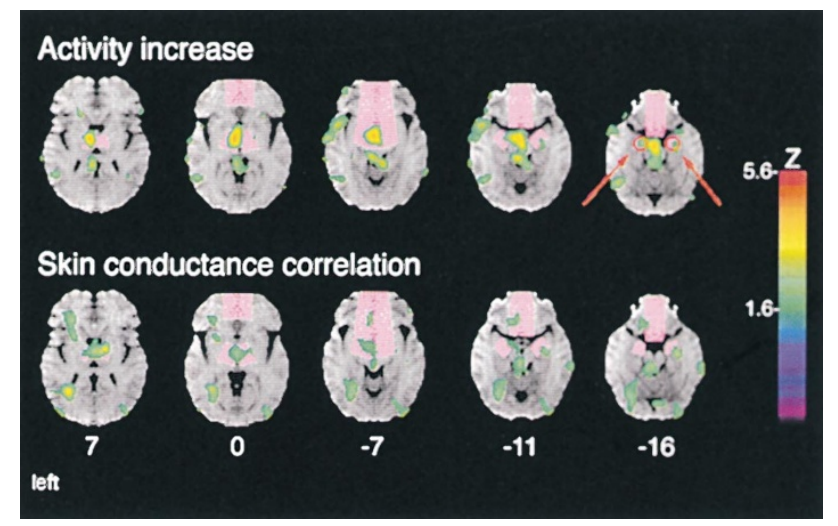

Figure 1. (A) Activity increase while viewing aversive stimuli occurs in limbic regions relative to viewing neutral pictures. The pink shading indicates the a priori search region; red arrows indicate the location of a spherical volume of interest for the amygdaloid complex. (B) Correlation map for activity and skin conductance, calculated on a voxel-by-voxel basis for all scan conditions, and Z-transformed. For this Figure as well as Figure 3, all Z-scores > 1.65 are displayed and superimposed upon a reference MRI scan. Numbers on the bottom row indicate $\mathrm{mm}$ above (or below) the bi-commissural line.

right middle frontal gyrus (Table 2). We did not note any trend level activations or decreases during this phase.

Interaction Effects. We examined the interaction between task and emotional valence by subtracting the effects of rating (negative minus neutral) from the effects recognition (negative minus neutral). We confirmed our prediction in the thalamic/hypothalamic region $(-1,3,-4 ; Z=3.95)$ and the right amygdala $(24,-8,-14$; $Z=3.85)$, both of which had greater activation for the aversive stimuli during the rating phase compared to the recognition phase. The VOI analysis confirmed this result in the right amygdala $(\mathrm{t}=2.95, \mathrm{df}=9, p<.01)$.

Our prior study had identified an interaction in the lingual gyrus, such that the effect of aversive stimuli was

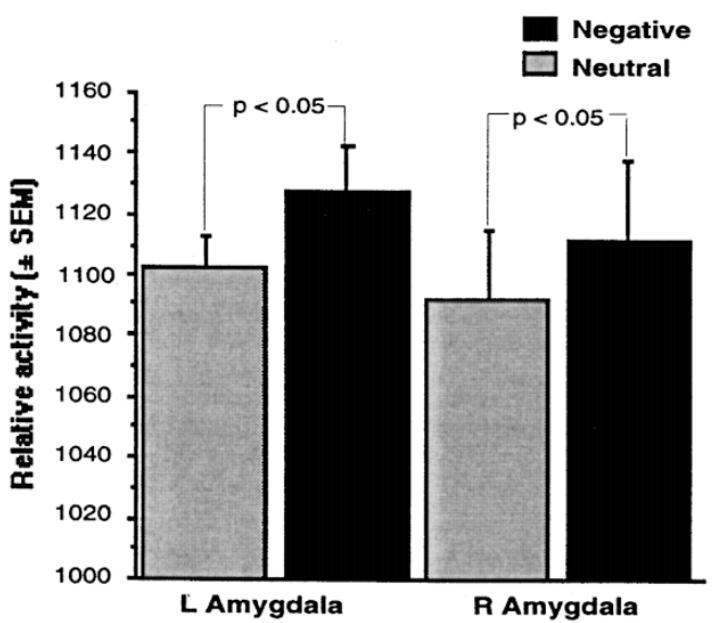

Figure 2. Activation of right and left amygdala during rating of pictures, normalized to mean of 1000 for gray-matter pixels. Activity calculated for spherical volume of interest of approximately $11 \mathrm{~mm}$ centered on amygdala coordinates derived from the Talairach and Tournoux (1988) atlas.

greater during the recognition phase. As Figure 3 indicates, a confluent area of activation from the lingual gyrus through the striate cortex confirmed this prediction, with a significant focus in the striate cortex $(1,-82,11 ; \mathrm{Z}=$ $-4.49)$, and a trend level focus near the interaction reported in our first study $(-10,-82,-9 ; Z=-3.52)$. Placement of the a priori VOI derived from this study showed a trend toward interaction $(\mathrm{t}=1.64, \mathrm{df}=9, p=.07)$.

Correlations. We examined voxel-by-voxel correlations between cerebral activity and the integrated SCR response. Within a priori defined regions, we found significant positive correlations between activity and skin conductance in the right thalamus $(15,-6,14, \mathrm{Z}=3.6)$ and trend level activations in the limbic diencephalon and midbrain area. As Figure 1 demonstrates, the correlation peaks overlapped the activation regions. There were no significant correlation peaks outside a priori de-

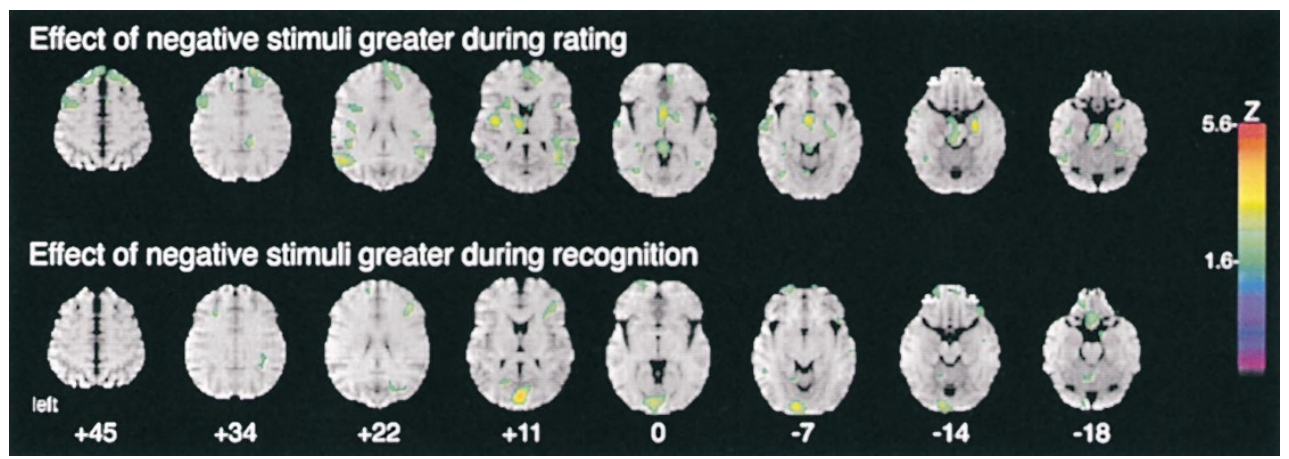

Figure 3. Effects of picture content interact with task. The top row shows the effects of negative emotional content (negative minus neutral) greater during rating than during recognition (the difference of two difference images). The bottom row demonstrates where the effect of negative content is greater during recognition, relative to rating. 
Table 2. Negative Pictures Minus Neutral Pictures During Each Phase

\begin{tabular}{|c|c|c|c|c|}
\hline \multirow[b]{2}{*}{ Region $^{a}$} & \multicolumn{2}{|c|}{ Rating phase } & \multicolumn{2}{|c|}{ Recognition phase } \\
\hline & $(x, y, z)$ & Z-score & $(x, y, z)$ & Z-score \\
\hline & & Relative increase & & \\
\hline L middle frontal g (44/9) & $-51,10,27$ & 4.12 & & \\
\hline $\mathrm{R}$ middle frontal $\mathrm{g}(46)$ & & & $39,28,18$ & 4.83 \\
\hline Diencephalon & $-6,-6,0$ & 4.50 & & \\
\hline Midbrain & $1,-8,-14$ & 4.13 & & \\
\hline & & Relative decrease & & \\
\hline Striate cortex & $1,-82,11$ & -6.24 & & \\
\hline L lingual g (18) & $-12,-82,-7$ & -4.20 & & \\
\hline Orbital g (11) & $1,23,-18$ & -4.09 & & \\
\hline L cerebellum & $-35,-60,-29$ & -4.04 & & \\
\hline
\end{tabular}

This and table 2 list maxima/minima of foci of significant difference. Activation foci are shown with $Z$-scores $>3.5$, for regions with a priori hypotheses, and $Z>4.0$, for regions without a priori hypotheses. The $\mathrm{x}, \mathrm{y}$ and $\mathrm{z}$ coordinates derive from the Talairach and Tournoux atlas (Talairach and Tournoux 1988).: right/ left, anterior/posterior and superior/inferior, respectively. The numbers in parentheses refer to topographic regions based on the Brodman area labels from the atlas.

fined regions (significance threshold of $p<.05$ corrected for multiple comparisons).

Since we had no significant effect of negative content on heart rate, we did not analyze correlation maps. Furthermore, heart rate decreased across the course of the scanning session, so that session effects on cerebral activity would seriously confound any observed correlations. We did not observe a session effect for SCR.

\section{DISCUSSION}

Aversive emotional content activated a network of limbic regions including thalamus, extended amygdala, and hypothalamus in our subjects. The subjective reports and the skin conductance data further confirmed that the activation reflected the emotional response to the aversive pictures. Significantly lower activity in the right amygdala and limbic diencephalon was observed when the cognitive task was altered, confirming our prediction that limbic activation would be modulated by a change in cognitive processing. Similar modulation of activity in limbic cortex (anterior cingulate cortex) was reported by Lane and colleagues (1997), when subjects switched from assessing the emotional impact to judging non-affective qualities of a picture. Directing attention to personal experience (self monitoring) employed, could explain the different limbic region that got activated in this study (anterior cingulate cortex vs. amygdaloid region).

Modulation of limbic activity observed in these studies may reflect mechanisms to suppress or modify emotional responses during the cognitive task. While "suppression" of emotions could conceivably occurs without a change in the underlying limbic activity, our findings suggest an active process that directly reduces limbic responses. It is important to note, however, that some of the observed difference could have been due to the repetition of some slides during the recognition phase and additional studies will be needed to separate the effects of modulation and the effects of repetition (see discussion below). Additional work will be required to determine if this modulation originates in another structure, active but not apparent in our study, which projects to the amygdaloid complex and diencephalon, such as the entorhinal cortex/hippocampal formation (LeDoux 1993; Phillips and LeDoux 1992), or anterior cingulate cortex (Lane et al. 1997). Alternatively, modulation could occur within one of the activated regions, such as the extended amygdala.

We found prefrontal activation during rating and recognition of emotional stimuli which lateralized with the task. The activation trend in left middle frontal region during rating corresponded very closely to a focus from our first study. In a combined analysis of both data sets (18 subjects), we found a significant activation focus $(Z=4.7)$ in this region during the rating phase for the aversive visual stimuli. Left middle frontal gyrus activation by emotional content during the perception of the stimuli may reflect the appraisal process (rating the pictures) for the aversive stimuli, or alternatively, the finding may represent a component of affect regulation by the lateral prefrontal cortex (Stuss and Benson 1986). Although experiments with surface EEG have suggested that aversive stimuli elicit right prefrontal cortical activity (Davidson 1998; Kalin et al. 1998; Tomarken et al. 1992), differences in methodology (blood flow versus brain electrical activity) or duration of emotional state (persistent vs. transient) could account for our somewhat different findings. During the recognition phase, subjects did activate the right middle frontal gyrus. The right prefrontal cortex has been repeatedly associated with recognition/retrieval from episodic memory (Tulving et al. 1994) and activation by 
aversive relative to neutral pictures may reflect the process of picture recognition. Since these prefrontal regions exhibited a different lateralized pattern similar in each task, they may be candidates for regions which modulate activity in the extended amygdala as discussed above.

Alternative interpretations of the decreased limbic activation during recognition have to be considered as well. Previous reports have noted desensitization in the amygdala across an experimental session (Breiter et al. 1996; Taylor et al. 1998). Our counterbalanced, ABBA design would have protected against the effects of desensitization over the experimental session, and we found no session effect when the first and the last "rating" scans were compared. It is possible that the repetition of some of the pictures during the recognition task might have caused habituation in the amygdala weakening the activation signal. It is not likely however, that this could explain all of the findings, since $50 \%$ of the pictures viewed during each recognition scan were still novel to the subject, and we also selected aversive pictures with particularly strong negative content to avoid desensitizing subjects. Furthermore, skin conductance increases that were present during the recognition of the negative pictures argue against general desensitization. Additional studies, e.g., presenting previously seen pictures during the rating phase, will be able to fully assess the potential contribution of habituation to the observed modulation.

We have also found evidence of an interaction between the emotional activation and visual recognition in the striate cortex and lingual gyrus, replicating prior findings of greater activation for the recognition phase, compared to the rating phase. The lingual gyrus carries out early cortical elaboration of visual stimuli, and therefore would have been recruited for the perception of the complex visual stimuli (Livingstone and Hubel 1988; Van Essen et al. 1992). We have suggested that interaction in the lingual gyrus represents an enhanced processing of emotionally-laden stimuli during recognition (Taylor et al. 1998). Others have also reported differential activity in the visual cortex in response to emotionally salient stimuli (Kosslyn et al. 1996; Lang et al. 1998; Paradiso et al. 1997; Reiman et al. 1997).

Although in our earlier study we did not find differential activation of these regions during the rating phase, in our current study we noted a relative decrease of activity in the striate cortex and lingual gyrus during rating. Taken together, these findings add to a growing body of data showing that early visual processing is subjected to significant modulatory influences, particularly with respect to selective processing (Corbetta 1993; Watanabe et al. 1998).

Limbic regions, such as the amygdaloid nuclei, send projections to the early visual cortex (Amaral and Price 1984) and may be one source of modulation observed in the visual cortex. It is also possible that the instructions to attend to a subjective internal state ("how disgusting the image is") during the rating phase diverted the subject's attention from external stimuli, thereby decreasing primary visual processing. The interaction pattern in lingual gyrus and striatal cortex could also have been contributed to by differences in visual processing between the tasks. While our slides were matched with respect to the number of pictures portraying faces and human figures, they were not matched on visual complexity, luminescence, or color scheme, which could have contributed to the larger activation in these regions. On the other hand, recognition of negative pictures was easier than recognition of neutral pictures, as our subjects reported that they recognized the negative pictures more readily. With easier recognition, one would generally expect less activation in these regions for negative stimuli (Grasby et al. 1994).

The correlation maps for cerebral activity and peripheral skin conductance provide direct evidence linking negative emotional responses, peripheral autonomic changes and specific CNS structures. Correlations occurred in the same diencephalic regions where we observed increased activity in response to the emotional pictures. The thalamic focus of correlation appeared in the region of the anterior nucleus of the thalamus, which exchanges projections with the anterior cingulate cortex; however, activity from medial thalamic nuclei, which exchange projections with the amygdala, might also have contributed to the correlation map in Figure 1. The closely proximate lateral hypothalamus, may have also contributed to the observed correlations (Jansen et al. 1995). While we cannot adequately resolve individual thalamic nuclei with this PET methodology, a focus centered over a limbic nucleus reinforces the face validity of the results. Mangina and Beuzeron-Mangina (1996) found that direct stimulation of the human amygdala elicited ipsilateral SCRs, however the difference between direct stimulation and the more "physiologic" method used in our study could account for the differences in laterality. Others reported that amygdaloid lesions abolish conditioning of the SCR by emotional stimuli, while sparing the skin conductance component of the orienting response (Bechara et al. 1995). While links between the emotional response and the SCR, and between limbic structures and emotional response, have been established, the current findings provide direct neuroimaging evidence linking emotions and the peripheral autonomic response with central structures in humans.

Our results suggest a number of interesting directions for future investigation. The current study included female subjects only, thus studies with males will have to confirm the generalizability of these results. We observed activation in both amygdalae, but the task specific interaction and the correlation with the 
SCR were observed predominantly on the right. Others have noted laterality (Mangina and Beuzeron-Mangina 1996) or differential activation in right vs. left amygdala (Morris 1996; Morris et al. 1998). Whereas it is possible that this merely reflects the low experimental power, it is also possible that activation thresholds or the time course differ for right and left amygdalae, or that they play differential roles in the modulation of emotions. There is a need to further define the role of left middle frontal gyrus in the perception of emotional stimuli, and the right middle frontal gyrus involvement in recognition. With that in mind, we believe that our current findings provide strong evidence for task specific activation of an emotional network within limbic regions, and direct evidence of limbic diencephalon involvement in the peripheral SCR to emotional stimuli.

\section{ACKNOWLEDGMENTS}

This work was supported by the Ann Arbor Veterans Administration Medical Center (IL) and National Alliance for Research in Schizophrenia and Depression (SFT). Thanks are due to David Badre for analysis of the psychophysiology data.

\section{REFERENCES}

Adolphs R, Tranel D, Damasio H, Damasio AR (1995): Fear and the human amygdala. J Neurosci 15:5879-5891

Alheid GF, Heimer L (1988): New perspectives in basal forebrain organization of special relevance for neuropsychiatric disorders: The striatopallidal, amygdaloid, and corticopetal components of substantia innominata. Neuroscience 27:1-39

Amaral DG, Price JL (1984): Amygdalo-cortical projections in the monkey (Macaca fascicularis). J Comp Neurol 230:465-496

Bechara A, Tranel D, Damasio H, Adolphs R, Rockland C, Damasio AR (1995): Double dissociation of conditioning and declarative knowledge relative to the amygdala and hippocampus in humans. Science 269:1115-1118

Breiter HC, Etcoff NL, Whalen PJ, Kennedy WA, Rauch SL, Buckner RL, Strauss MM, Hyman SE, Rosen BR (1996): Response and habituation of the human amygdala during visual processing of facial expression. Neuron 17:875-887

Cherry SR, Woods RP, Mazziotta JC (1993): Improved signal-to-noise in activation studies by exploiting the kinetics of oxygen-15-labeled water. J Cereb Blood Flow Metab 13:S714

Corbetta M (1993): Positron emission tomography as a tool to study human vision and attention. Proc Natl Acad Sci U S A 90:10901-10903

Davidson RJ (1998): Anterior electrophysiological asymmetries, emotion, and depression: Conceptual and methodological conundrums. Psychophysiology 35:607-614
Dolan RJ, Fletcher P, Morris J, Kapur N, Deakin JF, Frith CD (1996): Neural activation during covert processing of positive emotional facial expressions. Neuroimage 4:194200

Folkman S, Lazarus RS (1985): If it changes it must be a process: Study of emotion and coping during three stages of a college examination. J Pers Soc Psychol 48:150-170

Friston KJ, Frith CD, Liddle PF, Frackowiak RS (1991): Comparing functional (PET) images: The assessment of significant change. J Cereb Blood Flow Metab 11:690-699

Geen RG, Rakosky JJ (1973): Interpretations of observed aggression and their effect on GSR. J Exp Res Pers 6:280-292

Grasby PM, Frith CD, Friston KJ, Simpson J, Fletcher PC, Frackowiak RS, Dolan RJ (1994): A graded task approach to the functional mapping of brain areas implicated in auditory-verbal memory. Brain 117:1271-1282

Gur R, Alsop D, Glahn D, Petty R, Swanson C, Maldjian J, Turetsky B, Detre JJ, Gee J, Gur R (In press): An fMRI study of sex differences in regional activation to a verbal and a spatial task. Brain Lang

Haxby JV, Ungerleider LG, Horwitz B, Maisog JM, Rapoport SI, Grady CL (1996): Face encoding and recognition in the human brain. Proc Natl Acad Sci U S A 93:922-927

Irwin W, Davidson RJ, Lowe MJ, Mock BJ, Sorenson JA, Turski PA (1996): Human amygdala activation detected with echo-planar functional magnetic resonance imaging. Neuroreport 7:1765-1769

Isenberg N, Silbersweig D, Engelien A, Emmerich S, Malavade K, Beattie B, Leon AC, Stern E (1999): Linguistic threat activates the human amygdala. Proc Natl Acad Sci U S A 96:10456-10459

Jansen ASP, Nguyen XV, Karpitskly V, Mettenleiter TC, Loewy AD (1995): Central command neurons of the sympathetic nervous system: Basis of the fight-or-flight response. Science 270:644-646

Kalin NH, Larson C, Shelton SE, Davidson RJ (1998): Asymmetric frontal brain activity, cortisol, and behavior associated with fearful temperament in rhesus monkeys. Behav Neurosci 112:286-292

Kosslyn SM, Shin LM, Thompson WL, McNally RJ, Rauch SL, Pitman RK, Alpert NM (1996): Neural effects of visualizing and perceiving aversive stimuli: A PET investigation. Neuroreport 7:1569-1576

Kring AM, Gordon AH (1998): Sex differences in emotion: Expression, experience, and physiology. J Pers Soc Psychol 74:686-703

LaBar KS, Gatenby JC, Gore JC, LeDoux JE, Phelps EA (1998): Human amygdala activation during conditioned fear acquisition and extinction: A mixed-trial fMRI study. Neuron 20:937-945

LaBar KS, LeDoux JE, Spencer DD, Phelps EA (1995): Impaired fear conditioning following unilateral temporal lobectomy in humans. J Neurosci 15: 6846-6855

Lane RD, Fink GR, Chau PM, Dolan RJ (1997): Neural activation during selective attention to subjective emotional responses. Neuroreport 8:3969-3972

Lang PJ, Bradley MM, Fitzsimmons JR, Cuthbert BN, Scott JD, Moulder B, Nangia V (1998): Emotional arousal and activation of the visual cortex: An fMRI analysis. Psychophysiology 35:199-210 
Lang PJ, Greenwald MK (1988): The International Affective Picture System Standardization Procedure and Initial Group Results for Affective Judgments. Technical Report IA. Center for Research in Psychophysiology, University of Florida, Gainesville, FL

Lazarus RS (1984): On the primacy of cognition. Am Psychol 39:124-129

LeDoux JE (1986): The neurobiology of emotion. In LeDoux JE, Hirst W (eds), Mind and Brain. Cambridge, Cambridge University Press, pp 301-354

LeDoux JE (1993): Emotional memory systems in the brain. Behav Brain Res 58:69-79

Livingstone M, Hubel D (1988): Segregation of form, color, movement, and depth: Anatomy, physiology and perception. Science 240:740-749

Mangina CA, Beuzeron-Mangina JH (1996): Direct electrical stimulation of specific human brain structures and bilateral electrodermal activity. Int J Psychophysiol 22:1-8

McGaugh JL, Cahill L, Roozendaal B (1996): Involvement of the amygdala in memory storage: Interaction with other brain systems. Proc Natl Acad Sci U S A 93:13508-13514

Minoshima S, Berger KL, Lee KS, Mintun MA (1992): An automated method for rotational correction and centering of three-dimensional functional brain images. J Nucl Med 33:1579-1585

Minoshima S, Koeppe RA, Fessler JA, Mintun MA, Berger KL, Taylor SF, Kuhl DE (1993): Integrated and automated data analysis method for neuronal activation studies using [O-15]water PET quantification of brain function, tracer kinetics and image analysis in Brain PET. Akita, Elsevier, pp 409-415

Minoshima S, Koeppe RA, Frey KA, Kuhl DE (1994): Anatomic standardization: Linear scaling and nonlinear warping of functional brain images. J Nucl Med 35:1528-1537

Minoshima S, Morrow TJ, Koeppe RA, Casey KL (1995): The insular cortex in cerebral autonomic network. Hum Brain Mapp 1:166

Morris J (1996): A differential neural response in the human amygdala to fearful and happy facial expressions. Nature 383:812-815

Morris JS, Ohman A, Dolan RJ (1998): Conscious and unconscious emotional learning in the human amygdala. Nature 393:467-470

Morris JS, Ohman A, Dolan RJ (1999a): A subcortical pathway to the right amygdala mediating "unseen" fear. Proc Natl Acad Sci U S A 96:1680-1685

Morris JS, Scott SK, Dolan RJ (1999b): Saying it with feeling: neural responses to emotional vocalizations. Neuropsychologia 37:1155-1163

Paradiso S, Robinson RG, Andreasen NC, Downhill JE, Davidson RJ, Kirchner PT, Watkins GL, Ponto LLB, Hichwa RD (1997): Emotional activation of limbic circuitry in elderly normal subjects in a PET study. Am J Psychiatry 154:384-389
Phillips ML, Young AW, Senior C, Brammer M, Andrew C, Calder AJ, Bullmore ET, Perrett DI, Rowland D, Williams SCR, Gray JA, David AS (1997): A specific neural substrate for perceiving facial expressions of disgust. Nature 389:495-498

Phillips RG, LeDoux JE (1992): Differential contribution of amygdala and hippocampus to cued and contextual fear conditioning. Behav Neurosci 106:274-285

Reiman EM, Lane RD, Ahern GL, Schwartz GE, Davidson RJ, Friston KJ, Yun LS, Chen K (1997): Neuroanatomical correlates of externally and internally generated human emotion. Am J Psychiatry 154:918-925

Smith CA, Haynes KN, Lazarus RS, Pope LK (1993): In search of the "hot" cognitions: Attributions, appraisals, and their relation to emotion. J Pers Soc Psychol 65:916929

Spitzer RL, Williams JBW, Gibbon M, First MB (1988): Structured Clinical Interview of DSM-III-R. Biometrics Research Department. American Psychiatric Press, Washington DC

Stuss DT, Benson DF (1986): The Frontal Lobes. Raven Press, New York

Talairach J, Tournoux P (1988): A co-planar stereotaxic atlas of a human brain. Stuttgart, Germany, Thieme-Verlag

Taylor SF, Liberzon I, Fig LM, Decker LR, Minoshima S, Koeppe RA (1998): The effect of emotional content on visual recognition memory: A PET activation study. Neuroimage 8:188-197

Tomarken AJ, Davidson RJ, Wheeler RE, Doss RC (1992): Individual differences in anterior brain asymmetry and fundamental dimensions of emotion. J Pers Soc Psychol 62:676-687

Tulving E, Kapur S, Markowitsch HJ, Craik FIM, Habib R, Houle S (1994): Neuroanatomical correlates of retrieval in episodic memory: Auditory sentence recognition. Proc Natl Acad Sci U S A 91:2012-2015

Van Essen DC, Anderson CH, Felleman DJ (1992): Information processing in the primate visual system: An integrated systems perspective. Science 255:419-423

Venables PH, Martin I (1967): Skin resistance and skin potential. In Venables PH, Martin I (eds), A Manual of Psychophysiological Methods. Amsterdam, North Holland, pp 53-102

Whalen PJ, Rauch SL, Etcoff NL, McInerney SC, Lee MB, Jenike MA (1998): Masked presentations of emotional facial expressions modulate amygdala activity without explicit knowledge. J Neurosci 18:411

Watanabe T, Sasaki Y, Miyauchi S, Putz B, Fujimaki N, Nielsen M, Takino R, Miyakawa S (1998): Attention-regulated activity in human primary visual cortex. J Neurophysiol 79:2218-2221

Worsley KJ, Evans AC, Marrett S, Neelin P (1992): A threedimensional statistical analysis for CBF activation studies in human brain. J Cereb Blood Flow Metab 12:900-918 